\title{
Review
}

\section{The importance of advanced modalities in wound healing: a focus on the effects of electrical stimulation}

\author{
Hussain MA
}

Keywords chronic wounds, electrical stimulation, wound healing

For referencing Hussain MA. The importance of advanced modalities in wound healing: a focus on the effects of electrical stimulation. Wound Practice and Research 2020; 28(4):168-172.

DOI https://doi.org/10.33235/wpr.28.4.168-172

\section{Abstract}

Wounds or ulcers result from damage to the body's surfaces such as skin and mucous membranes. Once damaged, our body starts the repair process which, under normal conditions, is an organised process involving multiple steps, tissues, cells and their products. However, failure of the repair process to occur in a synchronised and timely manner leads to delayed healing. Wounds failing to heal or progress by 8 weeks are usually categorised as chronic wounds. Non-healing chronic wounds are challenging because, with the passage of time, they can affect a patient's health, their quality of life, their ability to work and subsequently their finances. Wounds that fail to heal are a significant burden on health system budgets worldwide.

Current routine management of wounds includes cleaning, debridement, applying wound dressings, compression bandages and antibiotics. However, non-healing wounds that fail to respond to these measures require advanced clinical methods, some of which have encouraging results. This review article discusses these advanced modalities, with a specific focus on electrical stimulation (ES) as an example of an emerging method being developed for the treatment of chronic wounds.

\section{Introduction}

The management of wounds is clinically very important as non-healing wounds can affect a patient's quality of life from various aspects, including wellbeing, finances and ability to

\section{Malik Asif Hussain}

MBBS, MAppSc (Research), IIWCC, PhD College of Medicine, University of Hail, Hail, Kingdom of Saudi Arabia

Email hussain_amc2010@yahoo.com and mh.hussain@uoh.edu.sa undertake work. In the USA alone, chronic wounds affect around six million people and put about a US\$20-25 billion burden on the health system ${ }^{1,2}$.

Normal wound healing involves multiple cellular and biochemical elements; the repair process is precisely controlled. However, in the case of delayed healing, this control is lost ${ }^{3,4}$. Known mechanisms that contribute to impaired healing include a prolonged inflammatory response, the formation of biofilm ${ }^{5}$, failure of re-epithelisation ${ }^{6}$, and an imbalance of micro-molecules such as growth factors and cytokines $^{7,8}$. In addition, the lack of immune system cells and their products result in less granulation tissue formation and epithelial repair ${ }^{9,10}$. The rate of healing is therefore unpredictable as it depends on multiple factors including age, wound size, wound depth, blood supply, nutrients, other illnesses and bacterial infection ${ }^{11}$. Vascular diseases that can lead to hypoxia also contribute to delayed healing ${ }^{12}$. As such, a depressed immune system, bacterial enzymes, bacterial biofilm and increasing antibiotic resistance play very important roles in disrupting the normal healing process ${ }^{13-15}$.

Routine wound management involves cleaning, debridement, application of wound dressings, compression bandages, pain management and the use of antibiotics (topical and systemic). However, the failure of wounds to heal routinely leads to the development of advanced treatment modalities; these are particularly important for patients who prefer to choose non-surgical options to enhance the speed of wound healing. Three categories of patients could potentially benefit from such treatment methods ${ }^{16}$ :

- Patients with wounds which fail to heal with standard treatment.

- Patients with medical conditions, such as diabetes mellitus, which can impair the healing process.

- Patients preferring to choose such modalities instead of surgical options. 
A few examples of advanced modalities include negative pressure wound therapy (NPWT), photodynamic therapy (PDT), topical negative pressure (TNP) dressings, low-level laser therapy (LLLT), ultrasound therapy and electrical stimulation (ES). This article focuses only on the potential use of ES for the treatment of impaired wounds.

More than 75 research and review articles have been studied to find related information for this article on ES. The main keywords used for the literature search were 'chronic wounds', 'wound healing' and 'electrical stimulation and wound healing'.

\section{Electrical stimulation in treatment of wounds}

Natural electrical fields exist in mammalians, amphibians and plants ${ }^{17}$. These fields are generated due to the movement of certain charged particles such as ions ${ }^{18}$. These electrical fields play important roles in normal tissue development, regeneration and repair of damaged tissues ${ }^{19,20}$.

Naturally-occurring electrical potentials occur over the skin; these have been demonstrated to be useful for enhancing proliferation of cells ${ }^{17}$. Thus, ES has been proposed as a useful modality that can be used to promote regeneration of tissues and healing ${ }^{16,21}$. Two types of stimulation methods are available. The first type uses monopolar energy delivered by electrodes, such as high-voltage pulsed current (HVPC) and low-intensity direct current (LIDC) ${ }^{22}$. This method is more effective than the second type of stimulation method which involves placement of bipolar electrodes - while the risk of infection is reduced in the bipolar approach as the electrodes are not transferred from one patient to another, the bipolar treatment is relatively slow in action compared to monopolar stimulation ${ }^{16}$.

The electrodes for ES are placed in the wound bed or over the skin near the wound area. A wet conductive medium such as normal saline soaked gauze or amorphous hydrogel impregnated gauze is usually used ${ }^{23}$ under the electrodes for current delivery to the wound bed or skin.

\section{Positive clinical outcomes}

Houghton and Campbell describe the positive effects of ES on cellular migration in the wound bed, including proliferation of cells, angiogenesis and re-epithelisation ${ }^{16}$. ES has also been shown to activate growth factors such as epidermal growth factor and to affect cellular movements in wounds ${ }^{24}$. Various mechanisms have been proposed to explain this migration of cells in the wound bed due to $\mathrm{ES}^{25,26}$. Nishimura et al. report movement of human keratinocytes under the influence of an electrical current $^{24}$ in an in vitro study. ES has also been shown to have positive effects on granulation tissue formation and proliferation, and can stimulate the remodelling process. ES causes synthesis of collagen and other proteins in the wound bed ${ }^{16}$. As explained above, the body's bioelectrical system plays an important role in the healing process. For instance, it enhances influx of the cellular elements which are involved in the repair process to the wound area. Furthermore, it influences the membrane permeability and release of secretions from the cells. It also affects the orientation of the cells which is important for a proper structural repair ${ }^{23}$. This system is also involved in a process known as 'galvanotaxis' which is the movement of cellular elements under the influence of endogenous electrical signals ${ }^{24}$. Further research is required, although ES is believed to mimic the effects of the body's bioelectrical system in promoting wound healing ${ }^{23}$.

The formation of new blood vessels (angiogenesis) is very important for successful wound healing. It ensures availability of nutrients and other materials that are required for cellular growth, proliferation and maturation ${ }^{27}$. Endothelial cells produce vascular endothelial growth factor (VEGF) after ES, which is involved in angiogenesis 27,28 .

It has been reported that treatment with ES decreases wound size and increases blood flow to the injured area ${ }^{29}$. One mechanism for increased blood flow is vasodilation which occurs due to stimulation of sensory nerve endings by $\mathrm{ES}^{30}$. Likewise, the formation of new blood vessels will also increase wound perfusion. The process of new blood vessel formation is enhanced under the influence of ES maturation ${ }^{27,28}$. The third reason for increased blood flow is linked with muscle pump activation which is explained later in this article.

Healing is enhanced by increasing the blood flow to the wound area. Importantly, increased blood flow, in conjunction with compromised or low drainage of blood, can cause oedema, which can hinder the healing process. This issue is usually tackled with various techniques such as physically activating the calf muscle by dorsiflexion and plantar flexion, elevating the wound area if possible, and using compression stockings and intermittent pneumatic compression (IPC) devices ${ }^{31,32}$. ES can address both of these effects simultaneously. It not only enhances the blood flow to the wound area but also activates the calf muscle which in turn improves blood drainage ${ }^{33,34}$. Figure 1 summarises the basic information related to ES technique, its mechanism of action, and its effects on wound healing.

ES improves wound healing by increasing perfusion to the wound area. Cosmo et al. report increased perfusion in wounds as well as surrounding areas during and after $\mathrm{ES}^{35}$. This means it causes dilation of vessels in the applied area. Similarly, increased success and survival of graft tissue has been reported if ES is applied locally. Increased perfusion also occurs due to new blood vessel formation angiogenesis ${ }^{36}$. Likewise, high levels of transcutaneous partial pressure of oxygen $\left(\mathrm{TcPO}_{2}\right)$ is observed in electrically stimulated wounds, which also indicates that blood flow and oxygen delivery to tissue is improved by $\mathrm{ES}^{37}$. 


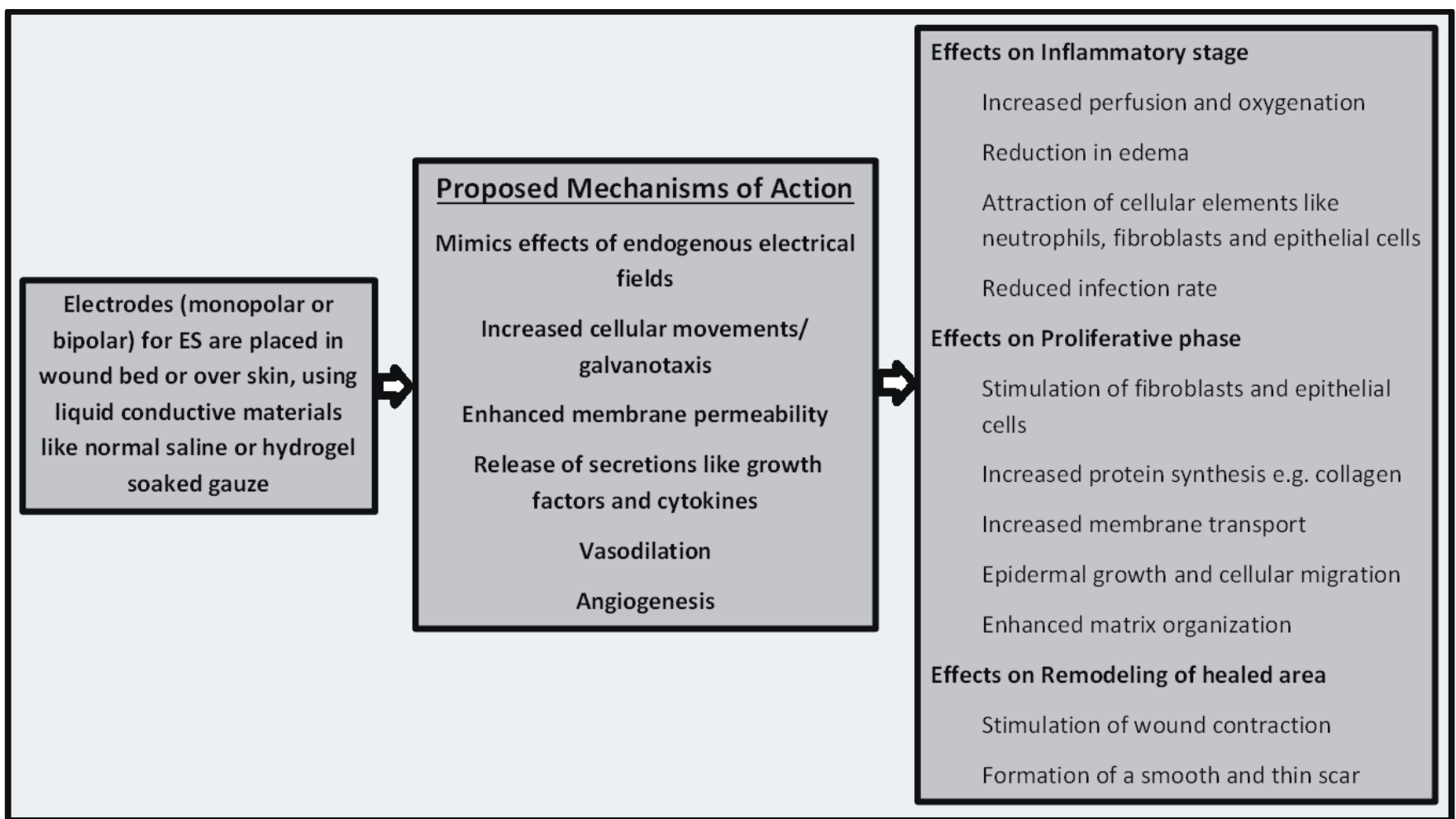

Figure 1. The technique, mechanism of action and effects of ES on the healing process

In normal physiological mechanisms, exercise increases lymph flow due to its effect on muscle contraction. This is the basis for the above described measure of activating the calf muscle by dorsiflexion and plantar flexion. A prominent example of ES for this purpose is the muscle pump activator (MPA). MPAs are devices which cause lower limb muscles to contract by stimulating the common peroneal nerve. MPAs improve healing by increasing blood flow, transcutaneous oxygen tension and venous return ${ }^{38-39}$. This makes MPAs a potential alternative to compression stockings. While stockings increase venous drainage, they may also reduce arterial flow due to application of pressure. MPAs increase arterial blood as well as venous flow ${ }^{38,40}$ and are reported to have better results in relation to healing outcomes, as well as patient satisfaction levels, compared to standard therapy with thrombo-embolic-deterrent (TED) and IPC devices ${ }^{40}$.

Wound infection is a major contributing factor to impaired healing. $\mathrm{Xu}$ et al. report that bacterial load and infection of wounds is reduced with ES use ${ }^{28}$. ES has also been shown to have positive adjuvant effects on antibacterial therapies. For example, silver ion penetration into the deeper tissues is increased in the presence of electrical signals, which increases its antibacterial activity ${ }^{21}$. Similarly, antibiotic effectiveness in removing biofilm has been reported to increase following ES treatment ${ }^{41}$. Studies have also shown that ES can have positive effects on the regeneration of axons of motor neurons during the healing process ${ }^{42}$. Interestingly, the scar quality and strength are also enhanced with ES treatment ${ }^{43}$.

\section{Clinical challenges}

The clinical challenges associated with ES include skin discomfort, irritation, tingling sensation and even skin burns. Thus, the therapy should be carefully monitored. Similarly, application of certain types of products or dressings can also influence the effectiveness of this treatment modality. For instance, petrolatum-based substances should not be used simultaneously with ES as they interfere with current conduction $^{16,23}$.

The amount of electrical current and its duration have not yet been fully standardised in wound care practice. Currently a $300-500 \mu \mathrm{C} / \mathrm{sec}$ charge is recommended for use; however, further evidence-based assessments of this technology are required ${ }^{21}$. Similarly, treatment duration and schedule vary considerably. For example, it can be applied a few times a week for a longer duration or can be applied daily for a shorter duration ${ }^{44}$. Standardisation of this approach is needed before its full potential can be fully realised for the treatment of wounds.

\section{Discussion}

ES is a potential therapy which can be particularly useful for wounds with delayed healing ${ }^{17}$. Research and clinical evidence is available to support its effectiveness and its use for the treatment of diabetic foot ulcers, pressure ulcers and venous ulcers. An analysis conducted on 22 randomised controlled trials reported the effectiveness of ES in promoting healing in various types of wounds ${ }^{45}$. Additionally, after reviewing 16 randomised clinical studies, Thakral et al. report 
that enhanced wound healing and damage area reduction was observed under the influence of $\mathrm{ES}^{46}$, something which has also been confirmed by other studies ${ }^{47-49}$.

It is clear from the above literature that ES enhances the mechanisms involved in the normal healing process. In our opinion, the clinical application of ES can play an important role in wound management. Furthermore, research evidence and availability of the technique are also very important factors to be considered. As treatment options are usually expensive, government subsidies or funding will be an important factor for the uptake of this technology.

Additionally, appropriate training in the use and delivery of ES would be required for its safe and effective use. If the technology is successful and cost effective, the patient demand is likely to increase. Without sufficiently trained physicians, the desired outcomes will not be achieved. Further research is needed to formulate an effective and improved treatment plan combining various therapeutic approaches.

\section{Conclusion}

ES is a useful technique which enhances natural healing mechanisms. Overall, ES appears to be a safe and effective option for the treatment of wounds. Skin irritation is the only major issue that has been reported. Both the duration time and the amount of current still need to be investigated in order to determine how these affect outcomes ${ }^{27}$. The current literature highlights the role of ES in wound healing but further research is required to address the parameters of duration, frequency, polarity, type and amount of ES. ES should also be investigated and standardised for use in all the various categories of wounds. Over the coming years it will be worth collecting further evidence to know the actual usefulness of ES, particularly in different types of wounds.

\section{Conflict of interest}

The authors declare no conflicts of interest.

\section{Funding}

The authors received no funding for this study.

\section{References}

1. Branski LK, Gauglitz GG, Herndon DN, Jeschke MG. A review of gene and stem cell therapy in cutaneous wound healing. Burns 2009;35(2):171-80.

2. Sen CK, Gordillo GM, Roy S, Kirsner R, Lambert L, Hunt TK, et al. Human skin wounds: a major and snowballing threat to public health and the economy. Wound Repair Regen 2009;17(6):76371.

3. Vistejnova L, Kralickova M. Foot ulcers in diabetes mellitus patients-protein analysis of wound environment. Paper presented at the EPMA Journal 2014; 5(Suppl 1):A74.

4. Berlanga-Acosta J, Schultz GS, Lopez-Mola E, Guillen-Nieto G, Garcia-Siverio M, Herrera-Martinez L. Glucose toxic effects on granulation tissue productive cells: the diabetics' impaired healing. BioMed Research Int 2013:256043.
5. Stephens P, Wall IB, Wilson MJ, Hill KE, Davies CE, Hill CM, et al. Anaerobic cocci populating the deep tissues of chronic wounds impair cellular wound healing responses in vitro. $\mathrm{Br} \mathrm{J}$ Dermatol 2003;148(3):456-66.

6. Gurjala AN, Geringer MR, Seth AK, Hong SJ, Smeltzer MS, Galiano RD, et al. Development of a novel, highly quantitative in vivo model for the study of biofilm-impaired cutaneous wound healing. Wound Repair Regen 2011;19(3):400-10.

7. Menke NB, Ward KR, Witten TM, Bonchev DG, Diegelmann RF. Impaired wound healing. Clinics Dermatol 2007;25(1):19-25.

8. Edwards R, Harding KG. Bacteria and wound healing. Current Op Infect Dis 2004;17(2):91-6.

9. Galkowska H, Wojewodzka U, Olszewski WL. Chemokines, cytokines, and growth factors in keratinocytes and dermal endothelial cells in the margin of chronic diabetic foot ulcers. Wound Repair Regen 2006;14(5):558-65.

10. Bhattacharya R, Xu F, Dong G, Li S, Tian C, Ponugoti B, et al. Effect of bacteria on the wound healing behavior of oral epithelial cells. PloS One 2014;9(2):e89475.

11. Zhao G, Usui ML, Underwood RA, Singh PK, James GA, Stewart $P S$, et al. Time course study of delayed wound healing in a biofilm-challenged diabetic mouse model. Wound Repair Regen 2012;20(3):342-52.

12. Dalton T, Dowd SE, Wolcott RD, Sun Y, Watters C, Griswold JA, et al. An in vivo polymicrobial biofilm wound infection model to study interspecies interactions. PloS One 2011;6(11):e27317.

13. Bayles KW. The biological role of death and lysis in biofilm development. Nature Rev Microbiol 2007;5(9):721-6.

14. Warriner R, Burrell R. Infection and the chronic wound: a focus on silver. Adv Skin Wound Care 2005;18 Suppl 1:2-12.

15. McCarty SM, Cochrane CA, Clegg PD, Percival SL. The role of endogenous and exogenous enzymes in chronic wounds: a focus on the implications of aberrant levels of both host and bacterial proteases in wound healing. Wound Repair Regen 2012;20(2):125-36.

16. Houghton PE, Campbell KE. Therapeutic modalities in the treatment of chronic recalcitrant wounds. In: Krasner DL, Rodeheaver GT, Sibbald RG, Woo KY, editors. Chronic wound care: a clinical source book for healthcare professionals. 5th ed. Malvern, PA: HMP Communications; 2012.

17. Tai G, Tai M, Zhao M. Electrically stimulated cell migration and its contribution to wound healing. Burns \& Trauma 2018;6(1).

18. Levin M. Large-scale biophysics: ion flows and regeneration. Trends Cell Biol 2007;17(6):261-70.

19. Foulds I, Barker A. Human skin battery potentials and their possible role in wound healing. Br J Dermatol 1983;109(5):51522.

20. Finger S, Piccolino M, Stahnisch FW. Alexander von Humboldt: galvanism, animal electricity, and self-experimentation part 2: the electric eel, animal electricity, and later years. J Hist Neurosci 2013;22(4):327-52.

21. Kloth L, Zhao M. Endogenous and exogenous electrical fields for wound healing. 2010. In McCulloch JM, Kloth L, editors. Wound healing: evidence-based management. Philadelphia, PA: F.A. Davis; 2010. pp. 450-513.

22. Newton RA, Karselis TC. Skin $\mathrm{pH}$ following high voltage pulsed galvanic stimulation. Physical Ther 1983;63(10):1593-6.

23. Sussman C. Electrical stimulation. Wound Care Information Network 2000;15:2007.

24. Nishimura KY, Isseroff RR, Nuccitelli R. Human keratinocytes migrate to the negative pole in direct current electric fields comparable to those measured in mammalian wounds. J Cell Sci 1996;109(Pt 1):199-207. 
25. Zhao M. Electrical fields in wound healing: an overriding signal that directs cell migration. Seminars Cell Development Biol 2009;20(6):674-82.

26. Pu J, McCaig CD, Cao L, Zhao Z, Segall JE, Zhao M. EGF receptor signalling is essential for electric-field-directed migration of breast cancer cells. J Cell Sci 2007;120(Pt 19):3395-403.

27. Zhao M, Bai H, Wang E, Forrester JV, McCaig CD. Electrical stimulation directly induces pre-angiogenic responses in vascular endothelial cells by signaling through VEGF receptors. J Cell Sci 2004;117(3):397-405.

28. Xu L, McLennan SV, Lo L, Natfaji A, Bolton T, Liu Y, et al. Bacterial load predicts healing rate in neuropathic diabetic foot ulcers. Diabetes Care 2007;30(2):378-80.

29. Kloth LC. Electrical stimulation technologies for wound healing. Adv Wound Care 2014;3(2):81-90.

30. Khalil Z, Jian L, Helme R. The use of non-invasive transcutaneous and acupuncture electrical stimulation techniques to modulate peripheral microcirculation. Prog Microcirc Res 1997;9:57-9.

31. Padberg Jr FT, Johnston MV, Sisto SA. Structured exercise improves calf muscle pump function in chronic venous insufficiency: a randomized trial. J Vasc Surg 2004;39(1):79-87.

32. O'Meara S, Cullum N, Nelson EA, Dumville JC. Compression for venous leg ulcers. Cochrane Database System Revs 2012(11).

33. Kaplan RE, Czyrny JJ, Fung TS, Unsworth JD, Hirsh J. Electrical foot stimulation and implications for the prevention of venous thromboembolic disease. Thrombosis Haemostasis 2002;88(2):200-4.

34. Moloney MC, Lyons GM, Breen P, Burke PE, Grace PA. Haemodynamic study examining the response of venous blood flow to electrical stimulation of the gastrocnemius muscle in patients with chronic venous disease. Eur J Vasc Endovasc Surg 2006;31(3):300-5.

35. Cosmo P, Svensson H, Bornmyr S, Wikstrom SO. Effects of transcutaneous nerve stimulation on the microcirculation in chronic leg ulcers. Scand J Plastic Recon Surgery Hand Surg 2000;34(1):61-4.

36. Patterson C, Runge MS. Therapeutic angiogenesis: the new electrophysiology? Circulation 1999;99(20):2614-6.

37. Mawson AR, Siddiqui FH, Connolly BJ, Sharp CJ, Stewart GW, Summer WR, et al. Effect of high voltage pulsed galvanic stimulation on sacral transcutaneous oxygen tension levels in the spinal cord injured. Paraplegia 1993;31(5):311-9.

38. Tucker A, Maass A, Bain D, Chen L, Azzam M, Dawson H, et al. Augmentation of venous, arterial and microvascular blood supply in the leg by isometric neuromuscular stimulation via the peroneal nerve. Int J Angiol 2010;19(1):e31.

39. Quigley F, Faris I. Transcutaneous oxygen tension measurements in the assessment of limb ischaemia. Clin Physiol 1991;11(4):31520.

40. Aquil S, Sharma H, Alharbi B, Pacoli K, Luke PP, Sener A. The impact of a muscle pump activator on incisional wound healing compared to standard stockings and compression devices in kidney and kidney-pancreas transplant recipients: a randomized, controlled trial. Canadian Urological Assoc J 2019;13(11):E341.

41. Costerton JW, Ellis B, Lam K, Johnson F, Khoury AE. Mechanism of electrical enhancement of efficacy of antibiotics in killing biofilm bacteria. Antimicrob Agents Chemo 1994;38(12):2803-9.

42. Brushart TM, Hoffman PN, Royall RM, Murinson BB, Witzel C, Gordon T. Electrical stimulation promotes motoneuron regeneration without increasing its speed or conditioning the neuron. J Neurosci 2002;22(15):6631-8.
43. Demir H, Balay H, Kirnap M. A comparative study of the effects of electrical stimulation and laser treatment on experimental wound healing in rats. J Rehab Res Develop 2004;41(2).

44. Feedar JA, Kloth LC, Gentzkow GD. Chronic dermal ulcer healing enhanced with monophasic pulsed electrical stimulation. Physical Ther 1991;71(9):639-49.

45. Fernandez-Chimeno M, Houghton P, Holey EE. Electrical stimulation for chronic wounds. Cochrane Database of Systematic Reviews 2004(1).

46. Thakral G, LaFontaine J, Najafi B, Talal TK, Kim P, Lavery LA. Electrical stimulation to accelerate wound healing. Diabetic Foot Ankle 2013;4(1):22081.

47. Ashrafi M, Alonso-Rasgado T, Baguneid M, Bayat A. The efficacy of electrical stimulation in lower extremity cutaneous wound healing: a systematic review. Experiment Dermatol 2017;26(2):171-8.

48. Houghton PE. Clinical trials involving biphasic pulsed current, microcurrent, and/or low-intensity direct current. Adv Wound Care 2014;3(2):166-83.

49. Khouri C, Kotzki S, Roustit M, Blaise S, Gueyffier F, Cracowski $\mathrm{J}-\mathrm{L}$. Hierarchical evaluation of electrical stimulation protocols for chronic wound healing: an effect size meta-analysis. Wound Repair Regen 2017;25(5):883-91. 